\title{
CrystEngComm
}

Check for updates

Cite this: CrystEngComm, 2021, 23 , 8159

Received 6th August 2021

Accepted 1st November 2021

DOI: $10.1039 /$ d1ce01034d

rsc.li/crystengcomm

\section{Protein crystallisation with air bubble templates: case of gas-liquid-solid interfaces}

\author{
Wenqing Tian, Chris Rielly and Huaiyu Yang (D)*
}

\begin{abstract}
Heterogeneous surfaces, such as solid particles, are known to efficiently increase nucleation rates during crystallisations from solution. In this work, air bubbles have been used to act as heterogeneous surfaces, to facilitate the critical nuclei formation of large protein molecules. Protein crystallisation experiments were performed using the hanging-drop method, to investigate the effects of concentrations of lysozyme and sodium chloride, and air bubbles on the nucleation of crystals. The introduction of an air bubble template resulted in an overall reduction in the nucleation induction time, over the majority of the experimental conditions. With air bubbles in the hanging droplets, the population density of the lysozyme crystals was up to 1.5 times higher than that in the droplets that contained no bubbles. For the studied experimental conditions, the mass yield was also found to increase by adding air bubbles into the droplet.
\end{abstract}

\section{Introduction}

With the growth of interest in protein drugs in the pharmaceutical industries, the crystallisation of proteins has gradually shifted focus to the manufacture of stable and more controllable products at a larger scale, instead of production of large single crystals for crystallography. One of the major advantages of protein crystallisation in biopharmaceuticals is the stability of the crystalline structure during drug formulation, storage and delivery. ${ }^{1,2}$ Moreover, these crystallisation processes provide products with higher purities and with potentially lower production costs. ${ }^{3}$

Several efforts have been made to improve protein crystallisations and one of the most successful methods is to use engineered surfaces as heteronucleants. ${ }^{4-6}$ Engineered surfaces allow control of the crystallisation to produce desired crystal sizes and polymorphs, without changing the operating conditions. Moreover, the nucleation process can be substantially accelerated via favourable surface-solute interactions, such as porous silicon, gold, polystyrene divinylbenzene particles. ${ }^{6-10}$ Porous materials with certain pore sizes, which are slightly larger than the diameters of proteins, were able to promote local supersaturation and induce nucleus formation. ${ }^{6}$ Langmuir-Blodgett (LB) nanotemplates and molecularly imprinted polymers (MIP) have been also proven to be effective to improve the crystal quality and to increase the growth rate of protein crystals. $^{11-13}$ However, concerns were raised about the availability of LB nanotemplates for larger industrial scale

Department of Chemical Engineering, Loughborough University, LE11 3RH, Loughborough, UK. E-mail: H.Yang3@lboro.ac.uk applications. Furthermore, MIP and LB nanotemplates can facilitate salt crystallisation in addition to proteins nucleation. ${ }^{14}$

In contrast to the use of a solid template, a crystallisation using a gas-liquid interface does not introduce external impurities and it is easy to separate the end product and the mother solution. In 1974, the German company Henkell and Co. developed a method to disperse gas bubbles in a supersaturation solution to accelerate the crystallisation of potassium bitartrate for sparkling wine production. ${ }^{15}$ Wohlgemuth et al. ${ }^{16}$ checked if the bubble surface itself acts as nucleation centre, for batch cooling crystallisation of dodecanedioic acid, by varying the gassing periods, gas compositions (pure air or saturated with solvent vapour) and bubble surface areas. For crystallisation of adipic acid, it was found that gassing induced nucleation and achieved a narrower crystal size, even when the gas was applied close to the supersaturation limit. ${ }^{17}$ In an L-alanine crystallisation in a MSMPR cascade, the dispersion of air bubbles into a slightly supersaturated solution, produced crystals with a smaller mean size and a narrower size distribution. ${ }^{18}$ The utilisation of micro-bubble techniques has also expanded into the research of organic compounds for polymorph control. The crystallisation of the $\beta$-form of glycine could be controlled by bubble injection $;{ }^{19}$ minimizing the bubble size reduced the required supersaturation for the generation of the $\beta$-form of glycine and inhibited the polymorphic transformation back to the $\alpha$-form. ${ }^{19}$ During the crystallisation of indomethacin in a water/IMC-EtOH system, the addition of $\mathrm{N}_{2}$ fine bubbles decreased the supersaturation needed to obtain a high-yield of the metastable polymorph $\alpha$-form. ${ }^{20}$ These studies at larger scale reported the influence of air 
bubbles on the crystallisation processes. However, the mechanism is still not fully understood, due to limited observation on the gas-liquid interfaces during the nucleation and crystal growth.

The objective of the present study is to investigate the relation between gas-liquid interfaces and protein crystallisation, including the influence on the nucleation and crystal growth. In this work, protein crystallisations of lysozyme by hanging-drop experiments were conducted at $\mathrm{pH}$ 4.2 and using a $0.1 \mathrm{M}$ sodium acetate buffer with and without air bubbles. The range of lysozyme concentrations investigated was between 50 to $100 \mathrm{mg} \mathrm{mL}^{-1}$ and $0.7 \mathrm{M}$ to 2 $\mathrm{M}$ sodium chloride solutions were used as precipitant to test the influence of gas bubbles over a wide spectrum of conditions. The crystallisation processes were observed under an optical microscope and comparisons were made between cases with (1) one or two air bubbles added into the crystallisation solution of the hanging droplets, and (2) droplets of the crystallisation solution with no air bubbles, under the same operating conditions. The nucleation behaviour was analysed, and population density and yield were compared for these cases.

\section{Experimental}

\subsection{Materials}

Hen egg white lysozyme ( $\sim 70000$ units per mg protein), sodium acetate (purity $>99 \%$ ), sodium chloride (purity $>$ 99.5\%), and glacial acetic acid (purity $>99.5 \%$ ) were purchased from Sigma-Aldrich and used without further purification. Buffer solution was prepared as $0.1 \mathrm{M}$ sodium acetate in deionized water at a $\mathrm{pH}$ of 4.2 adjusted by addition of acetic acid at the room temperature of $20^{\circ} \mathrm{C}$. The common literatures studying lysozyme crystallisation use buffer $\mathrm{pH}$ ranging from 4.0 to $4.8^{5,21-23}$ and $\mathrm{pH} 4.2$ was selected based on preliminary screening experiments. The selection was based on (1) sufficient experimental interval for distinguishing the influences of the air bubble (low supersaturation was preferable for heterogeneous nucleation); (2) limited experiment period before the disappearing of the air bubbles (bubbles were dissolved in a range of 24-72 h). Therefore, range of conditions were selected for nucleation occurrence (nucleation above $1 \mathrm{~h}$ and below $72 \mathrm{~h}$ ) with the heterogeneous bubble surface remaining in the solution. Lysozyme solutions with concentrations from 50 to $100 \mathrm{mg} \mathrm{mL}^{-1}$ were prepared by dissolving lysozyme in this sodium acetate buffer solution; $\mathrm{NaCl}$ solutions were prepared using the same method to reach a concentration range of $0.7 \mathrm{M}$ to $2 \mathrm{M}$ at room temperature of $20^{\circ} \mathrm{C}$. The protein concentration was verified by measuring the absorbance in the UV-vis spectrophotometer (Thermo Scientific ${ }^{\mathrm{TM}}$ Nanodrop One $\odot$ ) at $280 \mathrm{~nm}$ and the concentration was found to within $90 \%$ confidence level using mass extinction coefficient $\left(\varepsilon_{1 \%}\right)$ of $2.64 \mathrm{~mL} \mathrm{mg}^{-1} \mathrm{~cm}^{-1}$ for lysozyme. All solutions were filtered through a $0.2 \mu \mathrm{m}$ cellulose acetate filter and stored at $20{ }^{\circ} \mathrm{C}$ in the incubator for further experimentation.

\subsection{Method}

In the hanging drop experiments, $1 \mathrm{~mL}$ of precipitant buffer solution is put in the reservoir and a droplet of crystallisation solution containing $2 \mu \mathrm{L}$ of the same precipitant buffer solution and $2 \mu \mathrm{L}$ of protein buffer solution is placed on a cover slip, as shown in Fig. 1(a). The cover slip is carefully inverted above the reservoir and sealed by grease to ensure a closed system.

The screening experiments were performed in 24-well plates (ThermoScientific Nunclon Delta Surface) in a thermostatic incubator (VWR INCU-Line 150R) at 20.0 \pm 0.1 ${ }^{\circ} \mathrm{C}$. To study the static bubble effect on the protein crystallisation, the traditional hanging droplet was compared to the droplet injected with one air bubble, as shown in Fig. 1(b) and (c), respectively. The air bubble was injected by a pipette (SciPette $0.1-2.5 \mu \mathrm{L}$ ) when mixing the crystallisation droplet onto the cover slide. In addition, a second gas bubble was injected into some droplets after nucleation to gain further understanding of how the bubble impedes the crystal growth process; see Fig. 1(d). The hanging drops were observed under an optical microscope (GT Vision GTC-20 and ThermoFischer DXR Raman Microscope) at time intervals of hours or days based on the supersaturation. The induction time was determined by the first time when any visible crystal was observed in one droplet and used to quantitatively compare the nucleation process and influence of experiment conditions on the nucleation. Consequently, the error in observing the induction time is $\pm 0.5 \mathrm{~h}$ due to the minimum one-hour observation interval. Due to the possible large variation in induction time observation, each condition was repeated for at least 50 droplets to give a confident result of the average induction time.

The microscope images of the crystals were then analysed with ImageJ software (Wayne Rasband, National Institute of Health, USA) to count the number of crystals formed, to obtain crystal size distributions, and to determine the induction time when crystals were first observed inside the droplets, or to note that nucleation had not occurred. The
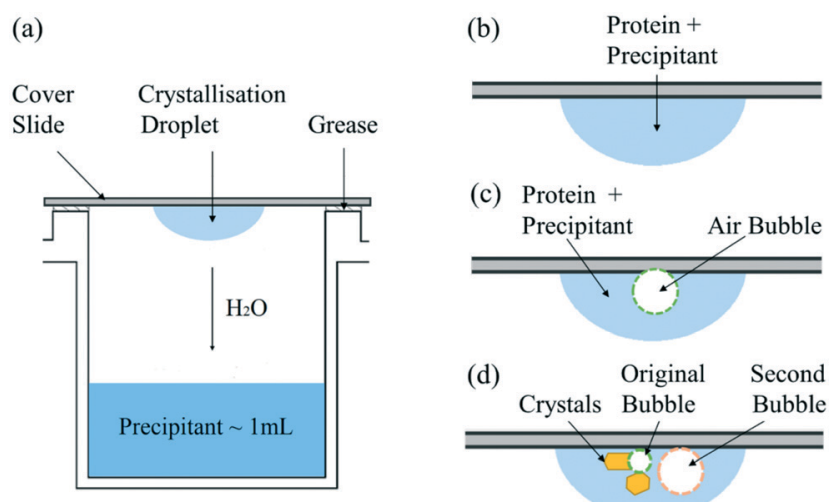

(c) Protein +

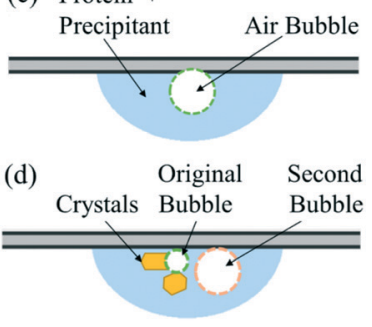

Fig. 1 Schematic diagram of (a) hanging drop experimental set-up; (b) droplet of crystallisation solution; (c) crystallisation solution with one air bubble injected; (d) crystallisation solution with second air bubble injected after nucleation. 
population density $(P)$, i.e., number of crystals per unit volume in the droplet was calculated to compare the influence of the air bubble on crystallisation progress. The area was measured by drawing the minimum diameter circle to enclose the crystals edges in the images, which was then converted into crystal size. The crystal size was estimated by using the circular equivalent diameter

$$
d=2 \times \sqrt{\frac{A}{\pi}}
$$

where $A$ is the area of the minimum diameter circle drawn as above. The mass yield $(\mathrm{m})$ in this experiment was calculated from the average crystal volume, the density of crystal, and the average population density. The volume of a crystal is estimated to be

$$
V=\frac{\pi d^{3}}{6}
$$

where $d$ is the diameter of crystal, based on the minimum circle which covers each whole crystal in the microscope images. The density of lysozyme $(\rho)$ was $1.2354 \mathrm{~g} \mathrm{~mL}^{-124}$ and hence the mass yield $(\mathrm{m})$ is calculated as

$$
m=V \times \rho \times P
$$

\section{Results}

As shown in Fig. 2 for bubble-free droplets, nucleation occurred earlier with an increase in lysozyme concentration and $\mathrm{NaCl}$ concentration. The mean induction time was significantly reduced when the lysozyme concentration increased to $100 \mathrm{mg} \mathrm{mL}$ with a constant $\mathrm{NaCl}$ concentration. For the same concentration of lysozyme, the induction time was significantly decreased when there was an increase in $\mathrm{NaCl}$ concentration up to $1.1 \mathrm{M}$. In the high

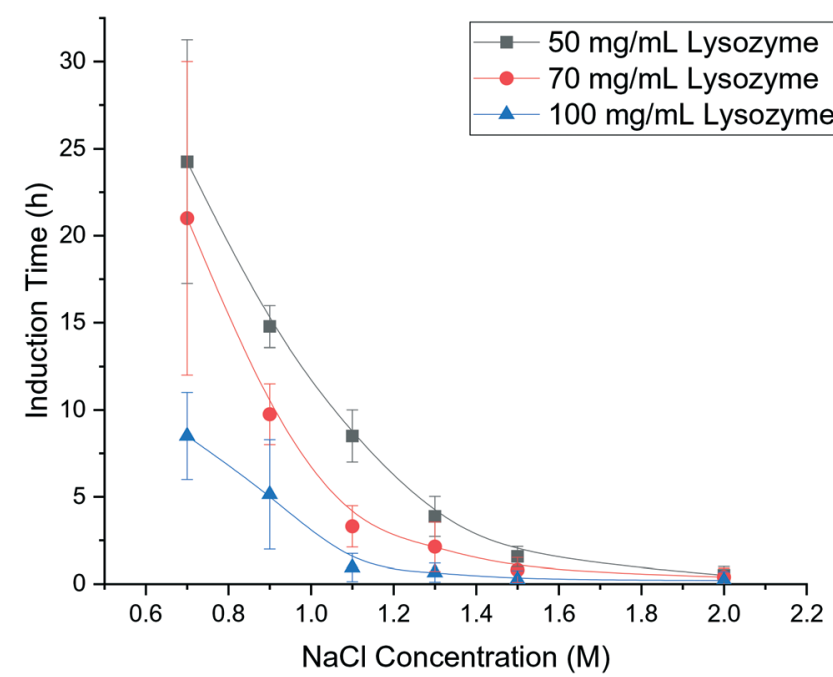

Fig. 2 Mean induction times for bubble-free droplets at different concentrations of lysozyme and $\mathrm{NaCl}$ and a fixed pH of 4.2. supersaturation region, $(\mathrm{NaCl}$ concentration above $1.1 \mathrm{M}$ or lysozyme concentration above $100 \mathrm{mg} \mathrm{mL}^{-1}$ ) there were reduced effects of the concentrations of lysozyme or $\mathrm{NaCl}$, and very short induction times were obtained under these conditions. Lin et l. $^{25}$ reported a similar dependence of the induction time on the reactants concentrations, in which a monotonic reduction in induction time was observed with an increase of the $\mathrm{NaCl}$ concentration or lysozyme concentrations, in agreement with the results shown in Fig. 2.

In the experiments with a single air bubble in the hanging drop, Fig. 3(a) shows that some crystals were attached to the bubble surface. Moreover, the crystal with a curved facet shown in Fig. 3(b) would appear to have nucleated and grown on the air bubble surface. Another explanation can be proposed for the curved facet: the critical nuclei first form in the bulk solution and then attach and grow on the air bubble. However, some bubbles were injected into the hanging drop after nucleation, and in these cases no crystals were observed with a similar curved shape. This result discounts the alternative explanation and indicates that it is highly probable that the lysozyme nucleates on the surface air bubble and continues to grow on the interface. Fig. 3(c) and (d) show that when the lysozyme nucleated on the bubble surface, the crystal would grow and move with the air-liquid interface. Fig. 3(c) shows two lysozyme crystals with length of $300-500 \mu \mathrm{m}$ which are growing on a bubble surface. When the air bubble shrank, both crystals were observed to rotate, dragged by the moving air-liquid surface. The movement was not because of the hydrodynamics of the solution in the droplets because all other crystals in the hanging drop remained in the same locations, as shown in Fig. 3(d). The crystal highlighted (inside the red circle of Fig. 3(c) and (d)) made a clockwise rotation, dragged by the bubble surface; the other crystal growing on the opposite side of the bubble underwent a similar rotation due to the shrinkage and translation of the bubble. All these phenomena showed that lysozyme crystals nucleate on the bubble, and the air-liquid surface acted as heterogeneous site for the nucleation. After nucleation, the crystals continued growing on the bubble surface and the bubble acted as a template, which can affect the shape of the crystals.

As shown in Fig. 1(c), experiments were conducted in which a second bubble (circled in orange in Fig. 4(b)) was injected into the droplet, after the visible formation of lysozyme crystals. In these experiments, the second bubbles were found to be less stable, with a much higher rate of shrinkage than the first bubble (circled in green) injected. Fig. 4(a) shows the droplet just before the injection of the second bubble. When the second bubble was injected, its diameter was about two times larger than the diameter of the original bubble, as shown in Fig. 4(b). After 21 hours, the second bubble shrank to become smaller than the first bubble, as shown in Fig. 4(c). The variation in the concentration of lysozyme is proposed as the cause of this 
(a)

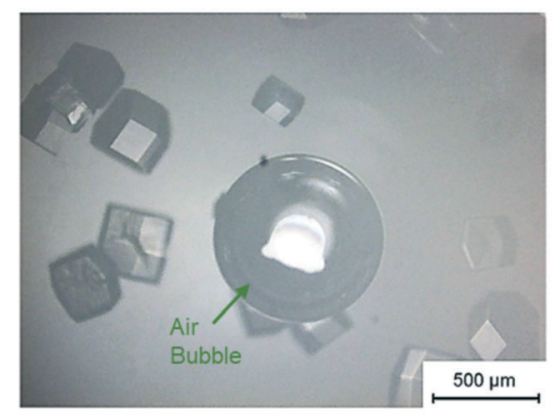

(c)

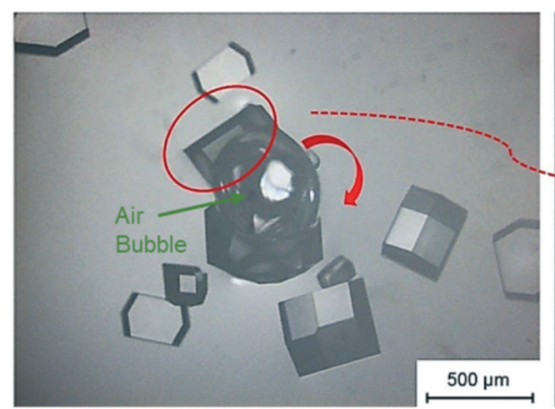

(b)

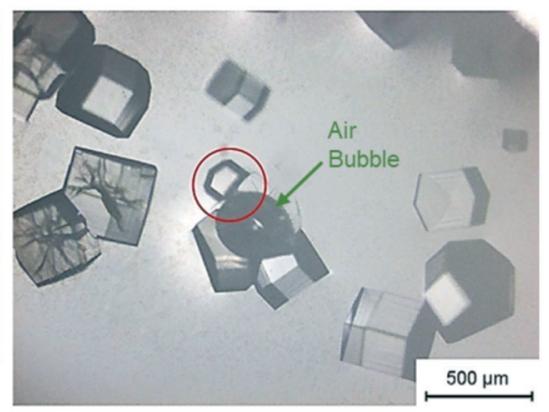

(d)

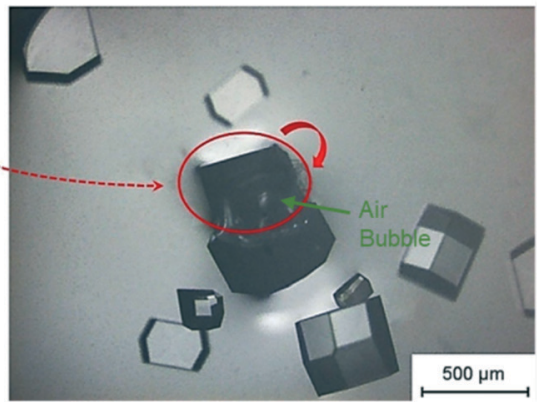

Fig. 3 Microscopic image of a crystal on bubble surface after 6 hours (a) and 55 hours (b) in a hanging drop with condition pH = 4.2, $1.1 \mathrm{M} \mathrm{NaCl,}$ and $50 \mathrm{mg} \mathrm{mL}^{-1}$ lysozyme and after 77 hours (c) and 140 hours (d) in a different hanging drop with condition $\mathrm{pH}=4.2,1.1 \mathrm{M} \mathrm{NaCl}$, and $70 \mathrm{mg}$ $\mathrm{mL}^{-1}$ lysozyme.

(a)

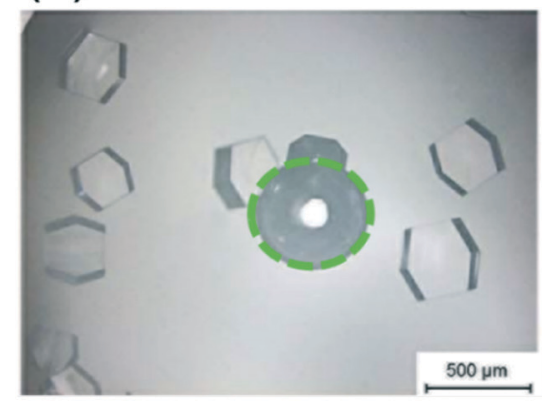

(b)

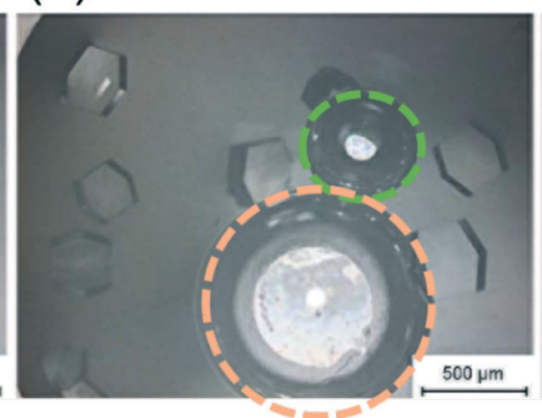

(c)

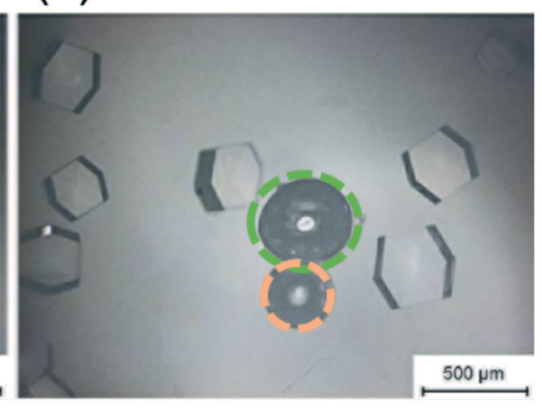

Fig. 4 Effect of second injected bubbles on crystallisation with condition $\mathrm{pH}=4.2,1.1 \mathrm{M} \mathrm{NaCl}$, and $50 \mathrm{mg} \mathrm{mL}^{-1}$ lysozyme: (a) just before the second bubble injection; (b) immediately after the second bubble injection; (c) 21 hours after the second bubble injection.

difference in bubble shrinkage rates. The protein acts as a surfactant to stabilise bubbles in solution, ${ }^{26}$ preferentially adsorbing at the gas-liquid interface; the first injected bubble was coated with a higher surface concentration of protein and remained stable in the solution. Crystal formation depletes the bulk lysozyme concentration in the droplet and at the time of second bubble injection the concentration in the droplet has reduced about $70 \%$, which results in a lower surface concentration of proteins on the second bubble injected. Therefore, the second bubble shrank at a higher rate and was prone to collapse in the solution due to its lower stability. This is in agreement with literature that the protein forms an adsorbed layer at the air-water interface, due to the presence of hydrophobic functional group in the lysozyme molecule. ${ }^{26}$ The adsorbed layer provides an increased resistance to mass transfer and hence reduces the bubble shrinkage rates.

In order to investigate the influence of bubble on the curvature formation of the crystals, the second air bubble was injected to come into contact with several existing crystals. No crystal with curvature shape was observed around the second air bubble after 21 hours Fig. 4(c). It shows that the curvature shape is not a result of the restriction of crystal growth due to the presence of the air bubble in the solution 
but is for the reason that the crystals nucleated on the air bubble surface (first bubble injected) and then the crystal growth was limited by the air bubble shapes.

The mean induction time was calculated for droplets containing bubbles, using the same method as for bubblefree droplets and was compared to the data presented in Fig. 2. The induction times obtained for the tested range of reactant concentration, were compared for the bubbled and non-bubbled cases using a contour plot in Fig. 5. In Fig. 5, the red region represents conditions where the mean induction time was shortened in bubbled droplets; blue region represents the mean induction time was prolonged in bubbled droplets; green region represents the conditions when the difference between bubbled droplets and nonbubbled is less than $5 \%$. It is clear that for droplets containing bubbles under almost all conditions, lysozyme nucleated faster or at similar times on average compared to non-bubbled droplets. Only 2 out of 18 tested conditions showed a longer mean induction time in droplets with air bubbles but in more than half of the conditions studied, the droplet containing a bubble nucleated in a shorter induction time. As shown in Fig. 5, the shortening in the mean induction time after injecting the air bubble was more predominant for lower lysozyme or $\mathrm{NaCl}$ concentrations. In fact, the greatest change in the mean induction time was found at the lowest concentration in this study: the average time for first crystals to appear in the droplets was shortened by $21 \%$ in the bubbled droplets for the experiment with 50 $\mathrm{mg} \mathrm{mL} \mathrm{m}^{-1}$ lysozyme and $0.7 \mathrm{M} \mathrm{NaCl}$ concentration (the lowest supersaturations studied). On the contrary, the introduction of a bubble had less impact on heterogeneous nucleation for the higher supersaturation conditions. Lin et $a l^{25}$ studied the nucleation kinetics of lysozyme crystallisation, and found that at lower supersaturations, crystals nucleated by a heterogeneous mechanism, whereas homogeneous nucleation occurred at higher supersaturations. Similarly in the current study at lower supersaturations, nuclei formed heterogeneously at the gas-liquid interface which lowered the surface free energy, and a shorter induction time was obtained after introduction of an air bubble.

Another potential reason for the above observations is that the high supersaturations, e.g., for $2 \mathrm{M} \mathrm{NaCl}$, often nucleate the crystal in less than $30 \mathrm{~min}$ (the typical time between images captured on the microscope) and it is relatively hard to quantify the difference between the induction times in this experimental set-up. Nonetheless, combining all the experimental conditions and repeat runs, the results indicate that with an air bubble present in the hanging drops, nucleation of protein crystals could occur more readily, with a shortening of the induction time up to $27 \%$ less than the non-bubble group.

The effect of air bubbles on protein crystallisation was also evaluated by analysing the crystal population density in the droplets with a range of lysozyme and $\mathrm{NaCl}$ concentrations under a $\mathrm{pH}$ of 4.2. In Fig. 6 the population density of bubbled and non-bubbled droplets is shown as red and blue columns, respectively by calculating the number of crystals per total volume of the crystallisation droplet. Noticeably, the population density was higher in the droplets with bubbles than droplets without bubbles in all tested conditions. In the case of $50 \mathrm{mg} \mathrm{mL}$ lysozyme, the population density of crystals in droplet with air bubble was about 1.5 times greater than the ones without bubbles. The enhancement of the population density in the bubbled droplet reduced as the lysozyme concentration increased, echoing the hypothesis of reduced influence of air bubbles for higher supersaturations in the study of nucleation. The trend was less obvious when comparing droplets with different $\mathrm{NaCl}$ concentrations. In the droplets with $1.1 \mathrm{M} \mathrm{NaCl}$ solution, the population density of the crystals in the droplet

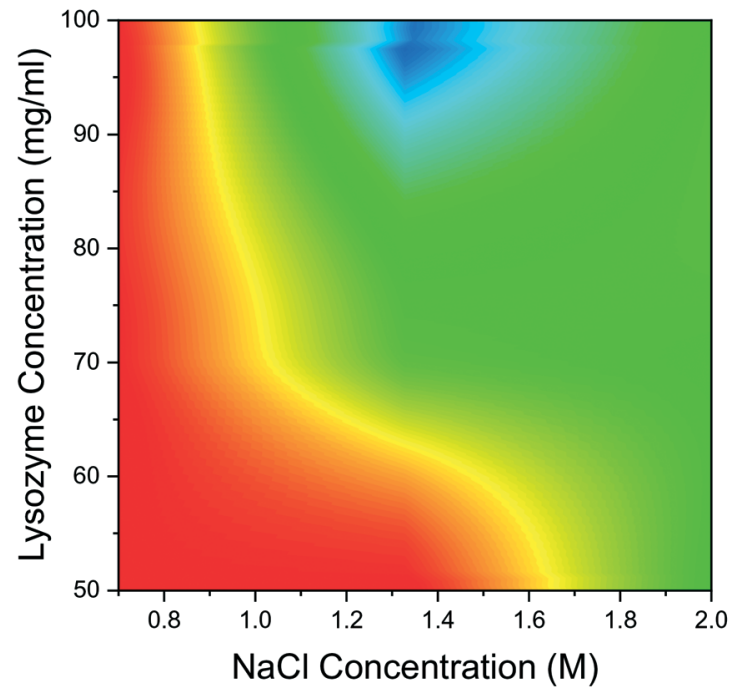

Comparison

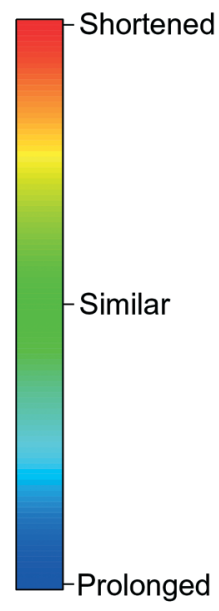

Fig. 5 Contour graph of mean induction times comparison between bubbled droplets and non-bubbled droplets for different conditions. 
a) $\mathrm{pH}=4.21 .1 \mathrm{M} \mathrm{NaCl}$

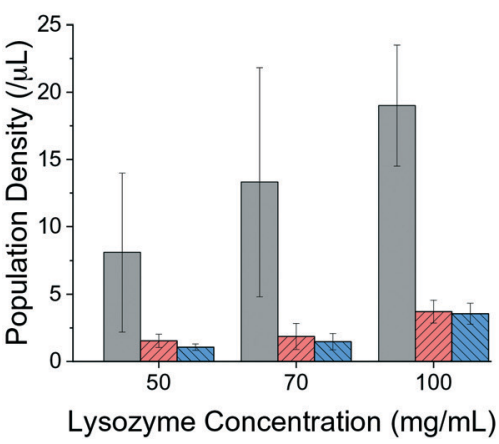

b) $\mathrm{pH}=4.270 \mathrm{mg} / \mathrm{mL}$ Lysozyme

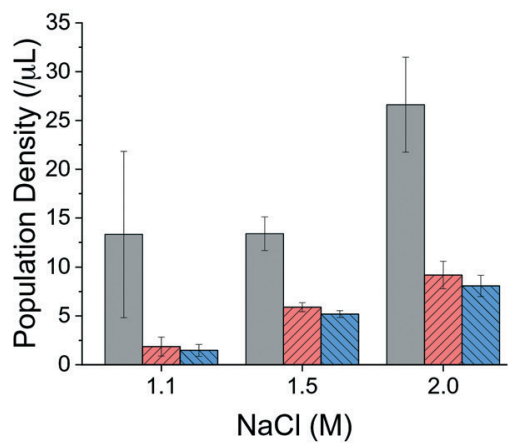

Droplet with one bubble:

Droplet without bubbles:
Solution around bubble Whole solution

Fig. 6 Population density comparison in different conditions for droplets with one bubble and without bubble.

with air bubble was about 1.3 times higher than for droplets without a bubble. At higher concentrations of $\mathrm{NaCl}$, the difference became less apparent.

The higher population density of crystals in the droplets with a bubble was mainly due to the large number of crystals attached to the bubble and located close to the bubble surface, indicating that gas-liquid interface facilitated nucleation and crystallisation. The average air bubble shrank $40 \% \pm 7 \%$, and the lower boundary condition $30 \%$ was used to cover most of the cases. A region, as 1.3 times larger than the diameter of the air bubbles at $26 \mathrm{~h}$ was selected to quantitatively compare the different locations in the hanging drop, as shown in the grey column in Fig. 6. The population density in the region near each bubble was obviously higher than the overall population density in the whole droplet. There were at least twice as many crystals around the bubble

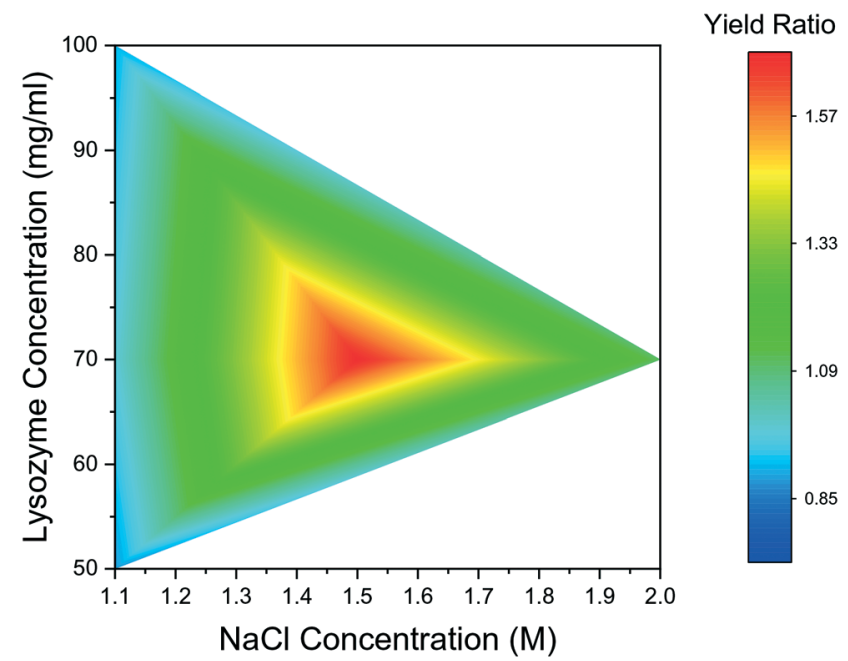

Fig. 7 Ratio of yields between droplets with one bubble and without bubble. than the other parts of solution in the hanging drop, under every experimental condition. As shown in Fig. 6(b), in the droplets with $70 \mathrm{mg} \mathrm{mL}^{-1}$ lysozyme, the population density of crystals was much higher in the solution near the bubble than the other parts of the solution in the droplet (by a factor of up to 7 times). In the high supersaturation conditions, such as $1.5 \mathrm{M}$ and $2 \mathrm{M} \mathrm{NaCl}$ concentrations, the increase of population density near the air bubble was smaller compared to cases with lower supersaturations.

Fig. 7 summarises yield comparison for bubbled and nonbubbled droplets at the aforementioned conditions. In Fig. 7, the ratio between mass yields of the bubbled droplets and bubble-free droplets are presented; if the ratio is greater than 1 , the bubbled droplets crystallised more lysozyme compared to non-bubbled droplets in the same period of time. The comparison result shows that crystal yields for droplets with one bubble were consistently greater than in droplets without bubbles across all conditions. For $50 \mathrm{mg} \mathrm{mL}^{-1}, 70 \mathrm{mg} \mathrm{mL}$, and $100 \mathrm{mg} \mathrm{mL}^{-1}$ lysozyme concentration respectively under $1.1 \mathrm{M} \mathrm{NaCl}$ concentration, the mass yields per unit solution volume were $16.2 \mathrm{mg} \mathrm{mL}^{-1}, 68.3 \mathrm{mg} \mathrm{mL}^{-1}$, and $95.8 \mathrm{mg} \mathrm{mL}^{-1}$ in the droplets with bubble, respectively. The percentage yield, defined as the ratio of crystallised mass to the theoretical supersaturated mass, was estimated to be $10 \%$ higher in the bubbled conditions than that in non-bubbled droplets at $70 \mathrm{mg} \mathrm{mL} \mathrm{m}^{-1}$ lysozyme concentration after 140 hours. The promotion effect of single air bubble on the crystal production was more apparent when the precipitant $\mathrm{NaCl}$ concentration increased and mass yields up to 1.8 times higher were achieved in the droplets containing an air bubble, compared to cases without bubbles at the same lysosome and $\mathrm{NaCl}$ concentrations. The general trend is that more lysozyme crystals formed over the same period of time in the droplets with an air bubble, due to faster nucleation and longer growth period, although this was not evident in every case. 


\section{Discussion}

The shrinkage of air bubble in the hanging drop was due to the air in the bubble dissolving in the solution. ${ }^{27,28}$ Under room temperature and atmospheric pressure, the air solubility in water is about $2.5 \times 10^{-5} \mathrm{~kg} \mathrm{~kg}^{-1}$ and a $4 \mu \mathrm{L}$ hanging droplet is able to absorb $10^{-10} \mathrm{~kg}$ of air if the solution is regarded as unsaturated with air. On the other hand, the average diameter of injected air bubble into the droplet was about $500 \mu \mathrm{m}$, which took about $7 \times 10^{-11} \mathrm{~m}^{3}$ in volume and $8.6 \times 10^{-11} \mathrm{~kg}$ in weight. In theory, the solution of the hanging droplet was able to dissolve this amount of air inside the air bubble. It is noted that the air could dissolve into solution through two air-liquid interfaces, i.e. inner air (air bubble)-liquid interface and outer air (atmosphere)-liquid interface. To assess the more favourable air-liquid interface for air to dissolve through, the Laplace pressure, defined as the pressure difference between inside and outside of a bubble, is considered. The Laplace pressure $\Delta P$ is a function of surface tension $\gamma$ and the radius of the sphere $r$ and can be calculated from

$$
\Delta P=P_{\text {inside }}-P_{\text {outside }}=\frac{2 \gamma}{r}
$$

There are two spheres present in the hanging drop set-up, one air bubble and a solution hemisphere. Given that two interfaces share the same solution, the ratio difference between the Laplace pressures of these two surfaces is the reverse ratio of their sphere radii, which was normally 3-5 times at the time of injection in this study. Furthermore, the gas solubility in the solution is governed by Henry's law:

$$
c=k P
$$

where $c$ is the concentration of dissolved gas, $k$ is the Henry's law constant, and $P$ is the partial pressure of the gas. Although the difference in surface pressure is relatively small between two interfaces, it still provides a driving force for solubility difference and possible air diffusion. Air in the bubble is thus more readily to be dissolved in the solution compared to the surrounding air due to its smaller diameter and higher excess pressure. The residence time of gas bubble and the induction time is an interplay process. However, the interface is proposed mainly to adsorb the protein molecules. With the shrinking, the adsorbed layer with shrink with the surface and even though it fully dissolved, the pre-aggregated protein molecules could still provide an advantage in nucleation.

The decrease in the induction times with an increase in the protein concentration or the precipitant concentration was in agreement with the literature, ${ }^{25,29}$ due to an increase in supersaturation. The decrease in the induction time with air bubble present in the droplet was consistent with the previous research on gas injection in other crystallisation systems, ${ }^{30-32}$ in which the heterogeneous surface decreased the energy barrier of the nucleation.
According to the heterogeneous nucleation theory, the relation between the change in the overall free energy required to form a critical nucleus $\Delta G_{\text {critial }}^{\text {hetero }}$ and the corresponding the free energy change for homogeneous nucleation $\Delta G_{\text {critial }}^{\text {homo }}$ could be represented ${ }^{33}$ by

$$
\Delta G_{\text {critial }}^{\text {hetero }}=\varphi \Delta G_{\text {critial }}^{\text {homo }}
$$

and the factor $\varphi$ can be expressed by

$$
\varphi=\frac{2-3 \cos \theta+\cos ^{3} \theta}{4}
$$

where the contact angle $\theta$ is the angle between the lysozyme molecules cluster and the foreign surface, as indicated in Fig. 8. It was reported that solid templates can accelerate the heterogeneous nucleation processes, such as with nanoporous gold particles, ${ }^{34}$ engineered glass surface ${ }^{6}$ and animal hairs. ${ }^{35}$ For a solid foreign surface, the factor $\varphi$ is smaller than one, indicating less energy is required to form a critical nucleus on foreign surface. Similar to the case on solid surface, the contact angle of protein cluster on the bubble surface is smaller than $180^{\circ}$ and, therefore, it can be predicted that a lower energy barrier for nucleation leads to a faster induction time of lysozyme with air-bubble templates. However, the exact value of the contact angle of lysozyme on air bubbles needs to be further investigated.

It is important to point out that there are multiple interfaces existing in hanging drop set-up, i.e. inner and outer air-liquid interfaces and solid-liquid interface between glass slide and the solution shown in Fig. 1(c). The water molecules continuously evaporated to leave the solution through the outer air-liquid interface, leading to a high concentration of lysozyme, and the gas molecules in the air bubble continuously dissolved into the solution through inner air-liquid interface, leading to a shrinkage of the air bubble. In the hanging drop set-up, the diffusion of water from top droplet to the reservoir is relatively slow, ${ }^{36}$ leading to continuous increase in supersaturation before nucleation. The inter air-liquid interface surface area was much less than the outer air-liquid interface surface, however, the crystal

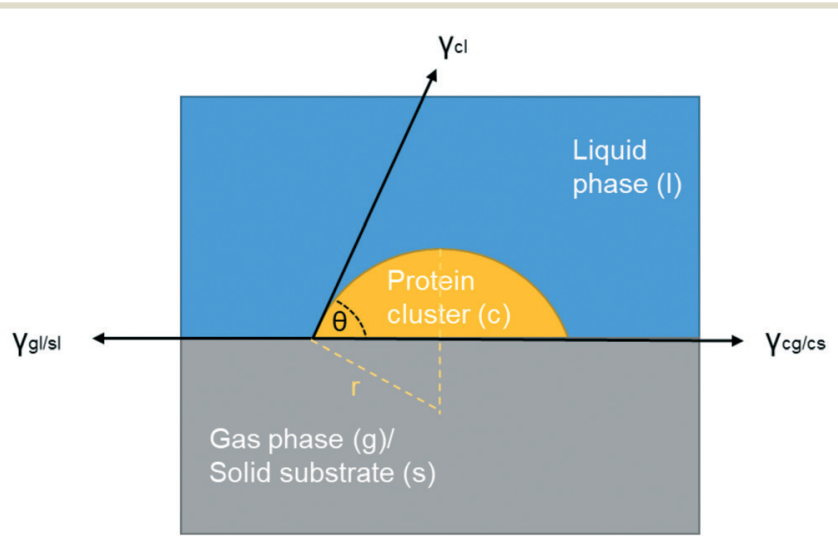

Fig. 8 Scheme of interfacial tensions at the boundaries when a foreign surface is present (gas or solid). 
morphologies and induction time results show the obvious influences of such small air-liquid interface, indicating the advantages of the inter air-liquid as a heterogeneous nucleation site and different mechanisms for the two air-liquid interfaces. For the solid-liquid interface, it has been revealed that the glass or functionalised silicon can enhance the nucleation of the protein, even though a pairing of the function group or the pore size with the hydrodynamic size of protein is required. ${ }^{37-40}$ However, both of the forementioned interfaces are present in both the bubbled group and the control group and this study focuses on the comparison and the difference between them. Same amount of the solution is pipetted onto the cover glass with more than 40 repeats, and as a result, the amount of solution contact with the cover slide and the outer vapour can be confidently assumed to be the same for the average result. The effect with the presence of these two interfaces can be eliminated by comparing the average results of the experimental and the control group and the discrepancy between these two groups have to arise from the addition of the air bubble, the only difference between them.

The curved surfaces formed on the protein crystal were very common in this study, such as examples shown in Fig. 3(b), indicating a strong interaction of lysozyme molecules and air bubble surface during the nucleation and crystal growth process. The polymorph of the curved crystals should be same as other crystals in the droplet, which all have tetragonal shape, but the growth of partly curved crystal was blocked by the air bubble template. Nonetheless, it is interesting that these crystals mostly formed on the interface between the injected air bubble and solution (inner air-liquid interface) but not on the interface between the hanging drop and air in the vessel (outer air-liquid interface). The shrinkage rate was different between these two air-liquid interfaces. The change in air bubble diameter after $26 \mathrm{~h}$ was about $40.0 \%$ (with standard deviation of $7 \%$ ) decrease compared to less than $0.5 \%$ (with standard deviation of $0.2 \%$ ) decrease for the solution droplet. Proteins can adsorb on the air-liquid interface acting as a lipid surfactant. ${ }^{26,41-44}$ The air bubble surface, adsorbing the proteins, had a multilayer structure, contributing to a slower desorption and, therefore, it was more difficult to achieve new dynamic equilibrium on the surface of the bubbles during the shrinking. ${ }^{45}$ The desorption rate may also be limited by the diffusion process. ${ }^{46}$ The lysozyme surface concentration was estimated to be $7.0 \mathrm{mg} \mathrm{m} \mathrm{m}^{-2}$ for the case of solution with $50 \mathrm{mg} \mathrm{\textrm {mL } ^ { - 1 }}$ lysozyme concentration, according to the literature. ${ }^{26}$ Assuming the amount of lysozyme molecules on the interface remained the same due to the slow desorption process, the surface concentration was calculated to be $20.0 \mathrm{mg} \mathrm{m}^{-2}$ at the inner air-liquid interface as the surface area reduced $65.0 \%$ in $26 \mathrm{~h}$ based on the $40 \%$ diameter decrease. Therefore, the concentration on the inner air-liquid interface was about three time higher than the concentration on the outer airliquid interface, due to limited changes in surface area of the outer air-liquid interface. Certain amount of lysozyme molecules adsorbed on both interfaces were able to desorb slowly (decrease in concentration), but it is reasonable to conclude that during the shrinkage of the air bubbles, the concentration on the inner air-liquid interface would always be higher than that on the outer air-liquid interface. The higher concentration, with higher supersaturation level near the air bubble surface area, would result in a thermodynamic advantage for the nucleation. ${ }^{47}$ Another possible explanation is differences in curvature of substrate surface for these two gas-liquid interfaces. Small bubbles (highly curved) have a higher excess pressure ${ }^{48}$ leading to topological formation, ${ }^{49}$ and minimisation of the curvature-induced elastic energy may attract more lysozyme molecules on/near the bubble surface. As mentioned before, the excess pressure of air bubble was about few times higher than the one of the droplets leading to higher chance in change of the topological formation. The higher accumulation of lysozyme molecules on air bubble-solution interface would accelerate the nucleation process. It was noted that population density in the solution immediately around bubble was always higher compared to the solution in the bulk of the droplet. The overall supersaturations in the hanging drop with or without bubble were assumed to be approximately equal at the same experimental time before the nucleation takes place. The temperature of the crystallisation solution in hanging drop was controlled as constant, and during the process, there were no differences of the droplet size observed with or without bubble, i.e., no differences in diffusion rate of water molecules from droplet with and without bubble to the reservoir. Although there were similar experimental conditions in the whole hanging drops with and without bubbles, the increase in the population density indicated that the air bubble in the hanging droplet changed the local thermodynamics or kinetics. However, compared with a solid template, an air bubble exhibits more complicated interactions with solution and solute, e.g., stabilisation of the air bubble by lysozyme molecules (similar to a surfactant), adsorption of lysozyme molecules on/near the air bubble surface, forming clusters on the air bubble interface, mass transfer and dissolution of air molecules into solution.

\section{Conclusion}

Crystallisation in hanging droplets with $0.7-2 \mathrm{M} \mathrm{NaCl}$ and 50-100 $\mathrm{mg} \mathrm{mL}^{-1}$ lysozyme was performed with one and two air bubbles and without an air bubble. The population densities of the crystals were increased up to 1.5 times in the droplets with an air bubble than the bubble-free droplets. During the same period of time, droplets containing one air bubble were able to produce $80 \%$ more lysozyme crystals in mass and could achieve almost theoretical yield in a shorter period time compared to the non-bubbled droplets. Moreover, the population density of the crystals in the solution near the bubble was up to nine times higher than the solution in the other parts of the droplets. Lysozyme can nucleate and grow on the air bubble surface, the inner air-liquid interface, demonstrated by the curved shape of the crystals and 
the rotational movements of the crystals during shrinking of the air bubble, indicating a strong interaction between the lysozyme crystal with the air-liquid interface. The crystallisation prefered to occure on the inner air-liquid interface than on the outer air liquid interface, probably due to the local supersaturation and the processes of the adsorption and desorption of lysozyme on the interfaces during shrinkage. Despite some variations, the air bubble overall accelerated the nucleation with most of the experimental conditions in this work. All the results suggest potential applications of air bubbles as soft templates in facilitating the protein crystallisation.

\section{Conflicts of interest}

There are no conflicts to declare.

\section{Acknowledgements}

WT would like to acknowledge school of AACME, Loughborough University, and $\mathrm{HY}$ is grateful to the UK EPSRC (Engineering and Physical Sciences Research Council) for support (EP/T005378/1).

\section{References}

1 C. Morrison, Fresh from the biotech pipeline-2016, Nat. Biotechnol., 2017, 35, 108-112.

2 A. Navarro, H. S. Wu and S. S. Wang, Engineering problems in protein crystallization, Sep. Purif. Technol., 2009, 68, 129-137.

3 H. Yang, W. Chen, P. Peczulis and J. Y. Y. Heng, Development and Workflow of a Continuous Protein Crystallization Process: A Case of Lysozyme, Cryst. Growth Des., 2019, 19, 983-991.

4 F. Artusio and R. Pisano, Surface-induced crystallization of pharmaceuticals and biopharmaceuticals: A review, Int. J. Pharm., 2018, 547, 190-208.

5 W. Chen, T. N. H. Cheng, L. F. Khaw, X. Li, H. Yang, J. Ouyang and J. Y. Y. Heng, Protein purification with nanoparticle-enhanced crystallisation, Sep. Purif. Technol., 2021, 255, 117384.

6 U. V. Shah, D. R. Williams and J. Y. Y. Heng, Selective Crystallization of Proteins Using Engineered Nanonucleants, Cryst. Growth Des., 2012, 12, 1362-1369.

7 N. E. Chayen, E. Saridakis, R. El-Bahar and Y. Nemirovsky, Porous silicon: An effective nucleationinducing material for protein crystallization, J. Mol. Biol., 2001, 312, 591-595.

8 N. E. Chayen, E. Saridakis and R. P. Sear, Experiment and theory for heterogeneous nucleation of protein crystals in a porous medium, Proc. Natl. Acad. Sci. U. S. A., 2006, 103, 597-601.

9 Y.-Z. Guo, L.-H. Sun, D. Oberthuer, C.-Y. Zhang, J.-Y. Shi, J.-L. Di, B.-L. Zhang, H.-L. Cao, Y.-M. Liu, J. Li, Q. Wang, H.-H. Huang, J. Liu, J.-M. Schulz, Q.-Y. Zhang, J.-L. Zhao, C. Betzel, J.-H. He and D.-C. Yin, Utilisation of adsorption and desorption for simultaneously improving protein crystallisation success rate and crystal quality, Sci. Rep., 2015, 4, 7308 .

10 U. V. Shah, J. V. Parambil, D. R. Williams and S. J. Hinder, Preparation and characterisation of $3 \mathrm{D}$ nanotemplates for protein crystallisation, Powder Technol., 2015, 282, 10-18.

11 E. Saridakis and N. E. Chayen, Imprinted polymers assisting protein crystallization, Trends Biotechnol., 2013, 31, 515-520.

12 D. R. Kryscio and N. A. Peppas, Critical review and perspective of macromolecularly imprinted polymers, Acta Biomater., 2012, 8, 461-473.

13 L. Belmonte, E. Pechkova, S. Tripathi, D. Scudieri and C. Nicolini, Langmuir-Blodgett nanotemplate and radiation resistance in protein crystals: state of the art, Crit. Rev. Eukaryotic Gene Expression, 2012, 22, 219-232, http://www. ncbi.nlm.nih.gov/pubmed/23140163 (accessed December 7, 2018).

14 R.-B. Zhou, H.-L. Cao, C.-Y. Zhang and D.-C. Yin, A review on recent advances for nucleants and nucleation in protein crystallization, CrystEngComm, 2017, 19, 1143-1155.

15 O. H. Rhein, Method for accelerating the crystallization and removal of tartar from a tartarous beverage, 1974, https:// patents.google.com/patent/US3988486A/en (accessed January 13, 2019).

16 K. Wohlgemuth, A. Kordylla, F. Ruether and G. Schembecker, Experimental study of the effect of bubbles on nucleation during batch cooling crystallization, Chem. Eng. Sci., 2009, 64, 4155-4163.

17 K. Wohlgemuth, F. Ruether and G. Schembecker, Sonocrystallization and crystallization with gassing of adipic acid, Chem. Eng. Sci., 2010, 65, 1016-1027.

18 M. C. Lührmann, M. Termühlen, J. Timmermann, G. Schembecker and K. Wohlgemuth, Induced nucleation by gassing and its monitoring for the design and operation of an MSMPR cascade, Chem. Eng. Sci., 2018, 192, 840-849.

19 M. Matsumoto, Y. Wada, A. Oonaka and K. Onoe, Polymorph control of glycine by antisolvent crystallization using nitrogen minute-bubbles, J. Cryst. Growth, 2013, 373, 73-77.

20 M. Matsumoto, M. Ohno, Y. Wada, T. Sato, M. Okada and T. Hiaki, Enhanced production of $\alpha$-form indomethacin using the antisolvent crystallization method assisted by N2 fine bubbles, J. Cryst. Growth, 2017, 469, 91-96.

21 H. Yang, P. Peczulis, P. Inguva, X. Li and J. Y. Y. Heng, Continuous protein crystallisation platform and process: Case of lysozyme, Chem. Eng. Res. Des., 2018, 136, 529-535.

22 J. Y. Y. Heng, X. Li, W. Chen, H. Yang and Z. Yang, Protein crystal occurrence domains in selective protein crystallisation for bio-separation, CrystEngComm, 2020.

23 E. L. Forsythe, R. A. Judge and M. L. Pusey, Tetragonal Chicken Egg White Lysozyme Solubility in Sodium Chloride Solutions, J. Chem. Eng. Data, 1999, 44, 637-640.

24 A. K. W. Leung, M. M. V. Park and D. W. Borhani, An improved method for protein crystal density measurements, J. Appl. Crystallogr., 1999, 32, 1006-1009.

25 C. Lin, Y. Zhang, J. J. Liu and X. Z. Wang, Study on nucleation kinetics of lysozyme crystallization, J. Cryst. Growth, 2017, 469, 59-64. 
26 V. S. Alahverdjieva, D. O. Grigoriev, J. K. Ferri, V. B. Fainerman, E. V. Aksenenko, M. E. Leser, M. Michel and R. Miller, Adsorption behaviour of hen egg-white lysozyme at the air/water interface, Colloids Surf., A, 2008, 323, 167-174.

27 N. F. Bunkin, S. O. Yurchenko, N. V. Suyazov and A. V. Shkirin, Structure of the nanobubble clusters of dissolved air in liquid media, J. Biol. Phys., 2012, 38, 121-152.

28 S. Michelin and E. Lauga, Collective dissolution of microscopic bubbles, APS, 2017, G7.007, https:/ui.adsabs.harvard.edu/ abs/2017APS..DFD.G7007M/abstract (accessed March 29, 2021).

29 N. E. Chayen, Turning protein crystallisation from an art into a science, Curr. Opin. Struct. Biol., 2004, 14, 577-583.

30 T. Kleetz, F. Funke, A. Sunderhaus, G. Schembecker and K. Wohlgemuth, Influence of Gassing Crystallization Parameters on Induction Time and Crystal Size Distribution, Cryst. Growth Des., 2016, 16, 6797-6803.

31 G. Capellades, S. Kiil, K. Dam-Johansen, M. J. Mealy, T. V. Christensen and A. S. Myerson, Effect of Air Injection on Nucleation Rates: An Approach from Induction Time Statistics, Cryst. Growth Des., 2017, 17, 3287-3294.

32 M. Matsumoto, Y. Morita, M. Yoshinaga, S. Hirose and K. Onoe, Reactive Crystallization of Lithium Carbonate Nanoparticles by Microwave Irradiation of Aqueous Solution Containing CO2 Microbubbles, J. Chem. Eng. Jpn., 2009, 42, s242-s248.

33 J. W. Mullin, Crystallization, Butterworth-Heinemann, 2001.

34 E. Pechkova and C. Nicolini, Accelerated protein crystal growth by protein thin film template, J. Cryst. Growth, 2001, 231, 599-602.

35 I. Nederlof, R. Hosseini, D. Georgieva, J. Luo, D. Li and J. P. Abrahams, A straightforward and robust method for introducing human hair as a nucleant into high throughput crystallization trials, Cryst. Growth Des., 2011, 11, 1170-1176.

36 A. McPherson and J. A. Gavira, Introduction to protein crystallization, Acta Crystallogr., Sect. F: Struct. Biol. Commun., 2014, 70, 2-20.

37 X. Tong, J. Kang, J. Zhang, X. Jia and W. Li, Interfacial functional terminals enhance the heterogeneous nucleation of lysozyme crystals, CrystEngComm, 2018, 20, 2499-2510.
38 D. Tsekova, S. Dimitrova and C. N. Nanev, Heterogeneous nucleation (and adhesion) of lysozyme crystals, J. Cryst. Growth, 1999, 196, 226-233.

39 M. M. Roberts, J. Y. Y. Heng and D. R. Williams, Protein crystallization by forced flow through glass capillaries: Enhanced lysozyme crystal growth, Cryst. Growth Des., 2010, 10, 1074-1083.

40 Y. X. Liu, X. J. Wang, J. Lu and C. B. Ching, Influence of the roughness, topography, and physicochemical properties of chemically modified surfaces on the heterogeneous nucleation of protein crystals, J. Phys. Chem. B, 2007, 111, 13971-13978.

41 B. Noskov and A. Mikhailovskaya, Adsorption kinetics of globular proteins and protein/surfactant complexes at the liquid-gas interface, Soft Matter, 2013, 9, 9392-9402.

42 E. Dickinson, R. Ettelaie, B. S. Murray and Z. Du, Kinetics of Disproportionation of Air Bubbles beneath a Planar AirWater Interface Stabilized by Food Proteins, J. Colloid Interface Sci., 2002, 252, 202-213.

43 R. Douillard and J. Lefebvre, Adsorption of proteins at the gas-liquid interface: Models for concentration and pressure isotherms, J. Colloid Interface Sci., 1990, 139, 488-499.

44 M. M. Ouberai, K. Xu and M. E. Welland, Effect of the interplay between protein and surface on the properties of adsorbed protein layers, Biomaterials, 2014, 35, 6157-6163.

45 R. Z. Guzman, R. G. Carbonell and P. K. Kilpatrick, The adsorption of proteins to gas-liquid interfaces, $J$. Colloid Interface Sci., 1986, 114, 536-547.

46 C. E. Morgan, C. J. W. Breward, I. M. Griffiths, P. D. Howell, J. Penfold, R. K. Thomas, I. Tucker, J. T. Petkov and J. R. P. Webster, Kinetics of Surfactant Desorption at an AirSolution Interface, Langmuir, 2012, 28, 17339-17348.

47 Y. Tsuchiya, Y. Wada, T. Hiaki, K. Onoe and M. Matsumoto, Effects of $\mathrm{CO} 2$ fine bubble injection on reactive crystallization of dolomite from concentrated brine, J. Cryst. Growth, 2017, 469, 36-41.

48 A. Cacciuto, S. Auer and D. Frenkei, Onset of heterogeneous crystal nucleation in colloidal suspensions, Nature, 2004, 428, 404-406.

49 G. Meng, J. Paulose, D. R. Nelson and V. N. Manoharan, Elastic instability of a crystal growing on a curved surface, Science, 2014, 343, 634-637. 